\title{
Is the Theoretical Unity of the Fallacies Possible?
}

\author{
JOHN WOODS University of Lethbridge
}

Key Words: Concept-in-use; exemplar model; Woods-Walton Approach; fallacy theory; logicísm; Lowenheim-Skolem theorem; neopythagoreanism; pragma-dialectic; reductionism; Stone representation theorem; summary representation; unification (one-way \& two-way).

\begin{abstract}
Historically, the fallacies have been neglected as objects of systematic study. Yet, since Hamblin's famous criticism of the state of fallacy theory, a substantial literature has been produced. A large portion of this literature is the work of Douglas Walton and John Woods. This paper will deal directly with the criticism of that work which has been advanced by van Eemeren and Grootendorst, particularly the complaints found in their writings of 1992, concerning the disunification of the fallacies and the exemplaristic approach of Woods and Walton's theories. It proposes a unification of the theories of Woods and Walton with that of van Eemeren and Grootendorst, and suggests that such a unification could be advantageous to both theories, and highly interesting for fallacy theory in general.
\end{abstract}

Considered as an object of systematic study, fallacies have had a long and undistinguished history. Ensuing from Aristotle's notion of paralogism, fallacies have met with recurring attention and little theoretical consensus. A millennial failure twiceover, it is a wonder that there hasn't been a more broadly based call for the abandonment of fallacy theory as a bad job. Not that there hasn't been some crankiness. Contemporary readers will think at once of Hamblin (1970), which scolds a long tradition for its lazy and uncritical subscription to a degenerate research programme. Still, Hamblin is no Churchland. He does not see himself as calling for the outright retirement of fallacy theory in that way that Churchland $(1981)^{1}$ calls for the abandonment of folk psychology. What Hamblin proposes is that we start doing fallacy theory right - to put it bluntly. What Churchland proposes is that the question of doing folk psychology right doesn't arise.

Harsher critics than Hamblin there surely are. One thinks of Lambert and Ulrich (1980) and Massey $(1975,1981)$ and their denial of the possibility of, or anyhow foreseeable prospects for, a bona fide theory of the fallacies. There is plenty of evidence that contemporary thinking has harkened to these objections. Writings on the fallacies do not routinely make their way into the leading journals. ${ }^{2}$ All the same, largely in response to Hamblin's challenge to reform the Standard Treatment, a substantial literature has been produced in the nearly quarter century since Fallacies appeared. It exists in quantities sufficient to sustain its own critical examination; and objections have come thick and fast. A goodly part of the post- 1970 record derives from the work, jointly and severally, of Douglas Walton and myself. For reasons that will shortly become apparent, and which I trust will not be thought to be too self-serving, much of what I have to say in this will focus on that body of work, in the abbreviating reference ' $W W$ '.

One complaint against WW is that it is too formal and context-insensitive (Finocchiario, 1987). Another is that although formal methods are admissible, 
WW sometimes does a bad job of motivating its own formal enthusiasms (Groarke, 1991). Another still is that the formalities do insufficient explanatory work. (Scriven, viva voce, the Third International Symposium on Informal Logic) and that they are an expository and analytical impediment (Scriven, again, and Groarke 1991). An especially interesting complaint comes from van Eemeren and Grootendorst in several writings, most recently in their book Argumentation, Communication and Fallacies (1992).

The systematic exploration of advanced

logical systems in order to analyze fallacies is characteristic of Woods and Walton's approaches (p. 103).

WW employs inductive logic in its treatment of secundum quid and post hoc; a plausibility logic underlies ad verecundiam; dialectical game theory is plumbed for many questions and petitio; a relatedness logic is pressed into service for ignoratio elenchi; and so on.

Every fallacy needs, so to speak, its own logic. For practical purposes this approach is not very realistic...One only gets fragmentary descriptions of the various fallacies... Ideally one unified theory that is capable of dealing with all the different phenomena is to be preferred (p. 103).

I want, in this chapter, to see if I can take the measure of this objection. ${ }^{3}$ Two questions press for attention. One is the question of what it is for a theory to be unified. The other concerns prospects for unifying fallacy theory in compliance with our answer to this prior question.

Talk of unifying theories puts us in mind of reductionism. Logicism is a case in point, the reduction of mathematics to set theory. Reductions of this sort interest philosophers largely for ontological reasons (though there is good reason to think that this was not the central interest of mathematicians). The reduction of chemistry to physics was considered important philosophically because it eliminated the ontological commitments of the reduced theory in favour of those of the reducing, or target, theory.
Philosophically-minded logicists felt the same way about the reduction of arithmetic to set theory. The basic idea is that for suitably constructed theories $\mathrm{T}$ and $\mathrm{T}^{*}$, if there exist mappings and definitions such that the theorems of $T$ are preserved in $T^{*}$ and the ontology of $T$ could consistently be considered as null under the preservation, then $T$ reduces to $T^{*}$. Concerning the reduction of Peano arithmetic to set theory, to Zermelo-Fraenkel's ZF say, all the laws of arithmetic come out true in an ontology of pure sets. By a careless convention, it is sometimes said that the reduction shows that numbers are sets. In fact, reductions do not in general validate so strong a claim as identity.

Logistic reduction presupposes the meeting of certain technical requirements. One is that the theories in question, both the to-be-reduced and the reducing, be construed as the deductive closure of sets of axioms. It is striking that where a theory is also first order and extensional, every true theory reduces to arithmetic, such is the providence of the Lowenheim-Skolem theorem in a strengthened version due to Hilbert and Bernays. Two things at once become apparent. One is that no theory announces itself a priori as the preferred reducing theory. So, for example, since set theory is true in the natural numbers and number theory is true in a universe of pure sets, the question of which, if either, we might favour as reducing must turn on considerations independent of the technical fact that the reduction can be brought off. Russell believed, at times, what many others wouldn't, that set theory is conceptually and ontologically "prior" to arithmetic. In that he may have been right, but if so, its rightness does not reside in the fact that arithmetic reduces to set theory. A second point is more striking still; perhaps it is also ontologically alarming. It is that precisely where reductions of the present kind are technically possible, neopythogoreanism threatens to be true. Whatever its intuitive subject matter, no true first 
order theory requires that anything exist other than the natural numbers.

Readers will have seen long ago that those who recommend the unification of fallacy theory cannot have logicism in mind as their model or paradigm. Still less do they counsel any course which eventuates in the fallacies being artifacts of arithmetic. There is no chance that fallacy theory will ever answer to the technical requirements of such reductions. If we have learned anything since 1970 it is this: The language of fallacy theory is not a first order language. ${ }^{4}$

\section{II}

Ask a first year university student what a fallacy is, and there is a good chance that he will answer along the following lines: A fallacy is a mistake like begging the question or asking someone if he has stopped beating his wife. Our respondent might also cite hasty generalization as a further example, and illustrate with "Cynthia is a bad driver; so women are bad drivers." If you pressed with "What is begging the question?", you might be told that it is the sort of thing you get when you reason that Jesus loves me, this I know, for the bible tells me so.

Suppose you asked for a characterization of hasty generalization. The answer might be that it is an induction from a single case. This is wrong of course. The Cynthia-case is a fallacious generalization and it is an induction from a single case. But not all single-case inductions are fallacious, as witness the fourteen-month-old toddler, who on reposing his hand on the element of his mother's stove learns in just one encounter "Never to do that again."

I assume that for fairly large populations, say the population of university undergraduates, fallacy is a concept-in-use, that is, a concept with which there is natural familiarity evidenced by conversational ease and independently of its articulation in a developed theory. Another way of saying this is that "fallacy" is a functioning item of the pre-scientific lexicon of this community. I also assume that this conceptin-use serves as a device of classification. Members of this conceptual community will have little difficulty in recognizing as a fallacy "VAT is a regressive tax, because VAT is a regressive tax." They might also be presumed to categorize it further as circular or question-begging. These assumptions are of a kind that one finds in psychological studies of concepts and their role in classification. ${ }^{5}$

What our imaginary case suggests (actually, it is far from imaginary - it is amply exemplified in the experience of lots of people I know) is that the concept-in-use of fallacy conforms to what psychologists know as the exemplar theory. If so, the concept 'fallacy' does not have a "summary representation". The concept of fallacy is not a unitary concept. This matters. Having rightly despaired of certain reductionist unifications for fallacy theory, it is natural to construe the unificationist challenge as calling for a treatment in which the unitary character of this concept of fallacy is explicitly recognized. But, as I say, there is reason to suppose that 'fallacy' is not a concept-in-use possessing a summary representation. In the psychological literature a concept $K$ is said to have a summary representation when there is a true sentence Ks are $\ddot{0}, \emptyset$, etc. ${ }^{6}$ of which the following three things are true.

(1) The representation is an abstraction from $\mathrm{K}$-phenomena.

(2) The representation underdescribes actual instances of $K$ (and so there will be K-things bearing features other $\ddot{O}, \emptyset$, etc.). (3) The representation is retrieved in situations of K-categorization (and so a possessor of the concept $K$ would, whenever she were presented with a test item, retrieve the summary representation, against which the test item would be compared)?

If the exemplar theory of concepts is correct in general, or if it is correct for the 
concept-in-use of fallacy, then fallacy will not, I say again, be a unitary concept. On the exemplar model, everyday concepts are represented by users by "separate descriptions of some of their exemplars" . For the concept bird, the associated representation might consist of the exemplars robin, bluejay, and sparrow, the sub-exemplar Carolina thrush, and the concrete instance "Fluffy", the concept-holder's pet canary. In our population of university students, the representation of the concept of fallacy is similarly configured. Exemplars such as begging the question and hasty generalization are mentioned, as are particular instantiations-the Cynthia generalization, the spouse-beating question, and the Jesusloves-me inference.

Consider now the mistake of characterizing an induction as the fallacy of hasty generalization when it is an induction from a single case. Experiments in cognitive psychology (e.g., Tversky, 1977) suggest a rather natural explanation of this kind of mistake. It is an explanation that takes seriously the exemplar theory of concepts and categorization. The error in question arises from retrieving a (concrete) exemplar - the Cynthia-case, for example - of which "mistaken induction from a single case" was a correct characterization, and generalizing on that characterization.

Our particular example briefly to one side, it is interesting and a trifle ironic that on the exemplar model of categorization it is predicted that categorizers will routinely be hasty generalizers. If this is right and if generalizations from single cases are always fallacious, then the exemplar theory will make of us routine fallacy-mongers in the bulk of our categorizations. Though there is some experimental evidence that we are less pure in our quotidian pursuits than we might have thought, it is too much to suppose that we get nearly everything wrong nearly all the time. At any rate, it is too much to suppose this in the absence of some hefty supplementary evidence that it is so. ${ }^{9}$
With that said, it is now apparent that if the target concept of fallacy theory is what I have been calling a concept-in-use, then we would expect a further three things to be true if the exemplar theory is correct in general or if it is correct for that conceptin-use. One is that the theory would be dominantly exemplary in its presentation of the fallacies. Another is that quite often the theory would correct standing or received characterizations of particular exemplars. That is, the theory would traffic in "theorems" (to speak tendentiously) in the form

(TH): So-and-so exemplar is not always (or ever) a fallacy.

Thirdly, the theory could be expected to attempt summary representations with regard to particular exemplars, but these would be motivated by TH disclosures and various other pronouncements of theory. For example, in light of the "theorem"

(THI); Not every induction from a single case is a fallacy.

it might be ventured that a generalization on an event $\mathrm{E}^{*}$ in the presence of a single correlation with an event $\mathrm{E}$ is justified by a risk-aversion strategy in those cases in which the costs attaching to recurrences of $\mathrm{E}^{*}$ are higher than the costs, if any, that attach to generalizing on it. Something like this fits our toddler case, but it clearly will not serve for women drivers, no matter what we might think of Cynthia.

It will not have escaped notice that the van Eemeren and Grootendorst (hereafter "VEG") characterization of WW places it squarely in the ambit of these three features. WW is highly exemplary; it quite often corrects for misgeneralizations from exemplars; and such summary representations as it aspires to typically do not precede theory but are consequent upon it. ${ }^{10}$ What I have been saying of late is worth repeating. It is that the disunification of the fallacies is nothing to complain of. It is precisely as things should be. Or it is if the exemplar theory is correct. 
It would be overdoing it to say that the correctness of the exemplar theory is by now a settled issue. No exemplar theorist sees it so. Exemplarism faces some nontrivial problems, ${ }^{11}$ but there is considerable evidence that it does better than its natural rivals, the classical account and the probabilistic account, both of which are unitary theories. ${ }^{12}$ To the extent that this is so, I conclude that if the challenge of VEG is to provide a framework in which the concept-in-use of fallacy is given unitary recognition, this is a challenge that cannot be met.

\section{III}

Rival views are possible, of course. The theorist might hold that fallacy, considered as a concept-in-use, is unfit for the austere flights of theory. Theories, as Quine reminds us, are free for the thinking up; they are expressions of our conceptual sovereignty. The concept-in-use of collection was of no help in the reduction of number theory; sets had to be contrived. Why should it not be the same way with fallacy? Concepts-in-use resist abundant and deep generalizations; so why not dispense with them? Why not stipulate something more streamlined, and anchor the stipulation in the theoretical fruitfulness that it abets? In asking these things, a further interpretation of the unificationist's challenge becomes apparent. It is now seen as the challenge to displace fragmented concepts-in-use with theoretically powerful stipulations. VEG itself is interpretable as a positive response to this challenge. Whatever the antecedent nuances of the concept-in-use of fallacy, fallacies are now any violation of the rules of critical discussion in contexts of conflict resolution. ${ }^{13}$ The rules, ten in all, are held to be conditions necessary and sufficient to fix the extension of the predicate "is a rational critical discussion". To the extent that violations of the rules are efficiently (not: effectively) recognizable, so too is the extension of the predicate "is a fallacy". It is all rather tidy, and fallacy is now as unitary a notion as the idea of a rule-violation of pragma-dialectics will allow.

If that were not progress enough, VEG proposes that the loose fragments of the concept-in-use need not be altogether given up on. They can be gathered up, unanalyzed (after all they are, as such, analytically recalcitrant) and given a place in the taxonomy of the stipulative theory. This is nicely set out in van Eemeren and Grootendorst (1992) at pages 212 to 217 , under the section heading "Traditional Fallacies as Violations of Rules For Critical Discussion". ${ }^{14}$ It is an opportune move. The pragma-dialectician can now say that his theory accounts for the traditional fallacies, never mind that it doesn't analyze them. ${ }^{15}$ Each "traditional" fallacy - begging the question, complex question, ad hominem, ad verecundiam, hasty generalization, the whole gang of eighteen as I called them in Woods (1992) - is pegged or triangulated by (a) the rule that it violates, (b) the stage of the critical discussion to which the rule applies, and (c) the party or parties to the discussion considered to be bound by the rule at that stage. Here is an example. Critical discussions come in stages, with various obligations on proponents of a thesis and those antagonistic to it to perform in certain ways at certain stages. The confrontation stage is the stage at which protagonist and antagonist acknowledge a disagreement, the resolution of which is the overall aim of the discussion. The object of the disagreement is called a "standpoint". Rule 1 pertains to this stage. It provides that "parties must not prevent each other from advancing standpoints or casting doubt upon standpoints" (p. 208). The traditional fallacy ad baculum is now represented as a violation, either by the protagonist or the antagonist, of rule $l$ at the confrontation stage. It is the violation of "putting pressure on the other party by threatening him with sanctions" (p. 2l2). 
In this way, each traditional fallacy is recognized in VEG. Each is given a characterization in VEG. But there is a difficulty. VEG's characterizations of the traditional fallacies are not much good. For example, the abusive ad hominem is said to consist in "doubting the expertise, intelligence, or good faith of the other party" (p. 212). This is certainly not in general true, apart from a stipulation that makes it so. In other cases, the description is vacuous, as with the fallacy of ambiguity, which is the "misus[e] of referential, syntactic or semantic ambiguity" (p. 212). This gives the appearance of a nasty setback. VEG's descriptions of the traditional fallacies are just as callow and half-baked as anything one would find in the Standard Treatment against which Hamblin protested to such good effect. With VEG and the Standard Treatment alike, the traditional fallacies come to the same thing. Even so, there is reason to think that, though they come to the same thing, they do not commit the same offense. For the Standard Treatment and its WW-like successors, too, it is essential that these descriptions be right. For a stipulator, like VEG, getting them right is optional. The fallacy in question is not the ad baculum or the abusive ad hominem or the fallacy of ambiguity. It is any violation of the rules in their application at appropriate stages. In the case of ad baculum, the fallacy is doing anything that prevents people from advancing or challenging starting points. If making threats is one way of doing that, then it is an instance of that fallacy. If VEG's description of doing that is a vacuous characterization of the ad baculum, who cares? The only point of bothering with it in the first place was to give a general and loose indication of its tentative membership in a class of cases that exemplify the fallacy of violating rule $l$ at the confrontational stage.

On the present interpretation of VEG as a stipulative rival of WW (and, of course, of the Standard Treatment too), VEG can afford being rather cavalier about their de- scriptions of the traditional fallacies. If VEG gets them right, well and good. If not, it hardly matters. For, as the man said, "the traditional fallacies don't pay the rent in pragma-dialectics". They are house-guests only. They inhabit VEG on sufferance.

\section{IV}

This, as I say, is the fate of the traditional fallacies on one natural interpretation of VEG's programme. If it were the correct interpretation, it would leave the would-be fallacy theorist with a perfectly tenable option. One would be to stick with 'fallacy' as a concept-in-use and to work away at it piecemeal in something like the WW way. The other would be to make a radical departure and to contrive a unified notion de novo in something like the VEG way. The rivalry would persist and victory, if it ever came, would depend on future developments in the service of antecedent presumptions about what a good result would consist in. But there is reason to think that this is not the correct interpretation of VEG. In fact,
...we think the kind of approach which
is carried out by Walton (and Woods) could be combined with a pragma-dia- lectical approach.... The combination could probably lead to a lot of progress in dealing with [outstanding problems]. (van Eemersen and Grootendorst 1989, p. 109.)

What is this combination? What would it look like? Would it constitute satisfactory fulfillment of yet another conception of unification?

In a celebrated paper, W.H. Stone followed up on some work of Tarski's and sought to unify logic and topology. Actually, he tried to unify Boolean algebra and topology, Tarski himself having established the link from logic to Boolean algebra. Like Tarski, Stone proposes a theory in which conjunction, alternation and negation correspond to (set theoretic) multiplication, 
addition and complementation. The closedopen sets under these operations form a Boolean algebra. Stone (1937) deals with a class of topological spaces, known as Boolean spaces, that display the following features. They are totally disconnected, compact Hausdorff spaces. A particular case of these is the Cantor space which is got by according the pair set $(0,1)$ the discrete topology and then assigning to the Cartesian product of countably many cases of it the product topology. The highlight of Stone (1937) is the Stone Representation Theorem. It establishes a duality between any Boolean algebra and some or other Boolean space. The Stone Representation Theorem is underivable in Boolean algebra and underivable in topology. It is got only from an arrangement that brings together and extends the two theories. It is a deep result. It identifies and characterizes certain structures which are inherently interesting for topology and Boolean algebra alike. It also facilitates the derivation of further results which provide new insights into each domain, with Boolean algebra illuminating the structure of Boolean space and Boolean space illuminating the structure of Boolean algebra where none of these results is derivable prior to unification. Stone's work unifies Boolean algebra and topology in the way that Descartes unified algebra and geometry. In particular, Descartes' analytic geometry made it possible to give a good account of cubic curves, something that couldn't be done previously.

I apologize to readers for whom this brief interlude with Stone's Representation Theorem is unhelpful. Fortunately, we can now leave the mathematics and characterize its importance more abstractly and in ways clear to all. So let us say, to begin with, that

(1) $R$ is a representation relation between a theory $T$ and a theory $T^{*}$ just in case there is a truth preserving interpretation of a nonempty subtheory of $\mathrm{T}$ in a nonempty subtheory of $T^{*}$, or vice versa.
Let us also put it that

(2) $\mathrm{X}$ is an $R$-extension of $\mathrm{Y}$ iff $\mathrm{R}$ is a representation relation on a proper subtheory $X$ of $X$ and a subtheory $y$ of $Y$ and there are theorems provable in $\mathrm{X}$ that are unprovable in $\mathrm{x}$ (or in $\mathrm{y}$ ).

With these things said, we have

(3) The two-way unification principle: For theories $T$ and $T^{*}$, there is a two way unification of them just in case $R$ is a representation relation between $T$ and $T^{*}$ and there exists an R-extension of $T$ and an $R$ extension of $T^{*}$.

Derivatively, a one-way unification of $\mathrm{T}$ and $\mathrm{T}^{*}$ would be an R-extension of $\mathrm{T}$ or an R-extension of $\mathrm{T}^{*}$, but not of both.

We now have a precise question to ask. When VEG proposes the combination of WW and VEG in quest of further progress in dealing with outstanding problems, could these remarks fairly bear an interpretation according to which they are seen as calling for at least a one-way unification of WW and VEG? I think not. For one thing, as our discussion of logicism and the Lowenheim-Skolem theorem made clear, representation relations that stand any chance of constituting R-extensions will turn on structural features of the theories on which they are defined. There is little reason to think that WW or VEG possess or could be made to induce mathematical structures that will carry such representation relations in any literal sense. But the basic idea is attractive, and we may think that it could be approximated to intuitively by theories such as WW and VEG. The basic idea is that essential to a unification's success is an embedding of one "area" in another in a way that produces new insights not achievable in either of the prior two. In this regard, the WW treatment of the petitio in Kripke's intuitionistic logic can be thought of as a one-way unification of petitio-theory in intuitionistic logic which produces new insights about the petitio but not necessarily new theorems in intuitionistic logic. ${ }^{16}$ The present idea gen- 
eralizes on this. Couldn't we try for at least a one-way theoretical unification between (all the various theories that constitute) WW and VEG? I lack the space to explore this possibility in detail. Even so, some things are fairly clear. We might think that WW treatments of the dialectical fallacies would be especially amenable to unification. In that regard, Walton (1991), a substantial and complex monograph on begging the question achieves, if only somewhat modestly, a unification with certain parts of VEG and in ways that facilitate some of Walton's own results.

Prospects are less encouraging, perhaps, for other fallacies, for certain of the statistical fallacies, or for the gambler's fallacy division or post hoc. But, bearing in mind that every mistake of reasoning that can be made solo, can be made in the company of others and can, with some artful contrivance, be made in our reasoned engagements with others, a certain openness of mind may be called for. Although it doesn't strike me as obvious that running what I think about hasty generalization through the sieve of pragmadialectics will prompt new insights into hasty generalization, it doesn't strike me as obvious that it couldn't. It is something that I would be prepared to consider and to try.

At the end of the day or, more realistically, the century, one thing will be clear. The more that WW theories can be made to unify, one by one, with VEG the less it will be likely that the overall pattern of unification will be one-way. For it seems to me that under unification enough, the idea that a fallacy is just a violation of a rule of critical discussion will surely yield to something deeper and more complex. If so, the unification of WW and VEG would be genuinely two-way. And that would be interesting.

\section{Notes}

Massey's reservations and the thorny idea of logical form in an Eemeren et. al., forthcoming.

2 There are welcome exceptions, of course. Philosophy and Rhetoric, Argumentation, and Informal Logic stand out for their receptiveness to work in fallacy theory. There are recent indications that good academic presses are also lightening up, as evidenced by Hansen and Pinto (1994) published by the Pennsylvania State University Press, and of Walton (1992), published by the Clarendon Press. See also Hansen (1990).

I shall not contrive a defence of WW's formalisms beyond those offered by Woods and Walton (1989), chapter 17 and Woods (1989). Earlier responses to the unification challenge can be found in Woods (1988) and Woods (1992).

- I note here my intention to return to a mathematical notion of unification in Section IV.

See, for example, Smith and Medin (1981).

We may note that 2 is not independent of 1 ; but let that pass.
7 Smith and Medin $(1981,23)$.

Ibid., p. 144.

9 Actually we have a choice. Either single-case inductions are always fallacious or the exemplar theory is false because it implies that single-case inductions aren't always fallacious. I won't take the time to debate the options in detail. Suffice it to say that the exemplar theory is too good to be disabled by a reductio whose conclusion, in any event, is independently called into question by cases such as the toddler.

10 I attempt in Woods (1992) a theory-driven summary representation of the concept of fallacy itself.

11 Smith and Medin (1981), chapter 7.

12 Ibid, chapter 3 , and chapters 4 to 6 , respectively.

13 van Eemeren and Grootendorst (1992): "Fallacies are analyzed as...incorrect discussion moves in which a discussion rule has been violated (p. 104)"'.

14 Idem: "We think that all the traditional categories have their proper place in our system". 
is Their own use of "analyzed" in the quotation cited in note 13 is a careless colecism, doing no damage in context.

16 See Woods and Walton (1989), chapter 10.

17 I said in section I that philosophers tend to be interested in logicistic and Lowenheim-Skolem reductions for ontological reasons and that mathematicians are otherwise attracted by them. We can now be more precise. Mathematicians were engaged by such reductions for the promise they gave of fulfilling the two-way unification principle of the present section. That promise was amply realized. Arithmetic flourished in the transfinite and set theory was driven up the full reach of the cumulative hierarchy and beyond.
In preparing this chapter I have benefited from fruitful discussions with Ronald Yoshida whose advise, always pointful, is also generously given. Research was supported in the first instance by a fellowship-in-residence at the Netherlands Institute of Advanced Study (NIAS) and Frans $\mathrm{H}$. van Eemeren was leader of my research group. I thank them for their encouragement. I also thank, for its generous support, the Social Sciences and Humanities Research Council of Canada. Frans yan Eemeren and Rob Grootendorst read the paper in penultimate form and were able to spare me the embarrassment of corrigible errors. The incorrigible errors are entirely my own.

\section{References}

Churchland, Paul M. (1981). "Eliminative Materialism and the Propositional Attitudes", Joumal of Philosophy, 78, 67-90; reprinted as chapter one of Churchland (1989).

Churchland, Paul M. (1989). The Neurocomputational Perspective, Cambridge, MA: MIT Press.

Eemeren, Frans H. van and Grootendorst, Rob (1989). "A Transition Stage in the Theory of Fallacies", Journal of Pragmatics, 13, 99-109.

Eemeren, Frans H. van and Grootendorst, Rob (1992). Argumentation Communication, and Fallacies, Hillsdale, NJ, Erlbaum.

Eemeren, Frans H. van, Grootendorst, Rob, Blair, J. Anthony, Johnson, Ralph H., Willard, Charles A., Walton, Douglas N., and Woods, John, forthcoming. Fundamentals of Argumentation Theory: Classical Backgrounds and Modem Developments.

Finocchiaro, Maurice A. (1989). "Six Types of Fallaciousness", Argumentation, 1, 263-282.

Groarke, Leo (1991). Critical Review of Woods and Walton (1989), Informal Logic, Vol. XIII, No. $2,99-112$.

Hamblin, C.L. (1970). Fallacies, London: Methuen.

Hansen, Hans V. (1990). "An Informal Logic Bibliography”, Informal Logic, Vol. XII, No. 3, 154184.

Hansen, Hans V. and Pinto, Robert (eds.) (1994). Fallacies: The Dark Side of Reasoning, University Park, PA: The Pennsylvania State University Press.

Lambert, K. and Ulrich, W. (1980). The Nature of Argument, New York: Macmillan.

Massey, Gerald (1975). "Are There Any Good Arguments that Bad Arguments are Bad?", Philosophy in Context, 4, 61-77.
Massey, Gerald (1981). "The Fallacy Behind Fallacies", Midwest Studies in Philosophy, 6, 489-500.

Smith, Edward E. and Medin, Douglas L. (1981). Categories and Concepts, Cambridge, MA: Harvard University Press.

Stone, W. H. (1937). "Applications of the Theory of Boolean Rings to General Topology", Transactions of the American Mathematical Societ, 41,375-48I.

Tversky, A. (1977). "Feature of Similarity", Psychological Review, 84, 327-352.

Walton, Douglas N. (1991). Begging the Question: Circular Reasoning as a Tactic of Argumentation, New York: Greenwood Press.

Walton, Douglas N. (1992). Slippery Slope Arguments, Oxford: Clarendon Press.

Woods, John (1989). "The Necessity of Formalism in Informal Logic", Argumentation, 3, 149-167.

Woods, John (1988), "Pragma-Dialectics: A Radical Departure in Fallacy Theory", ISSA News. letter (December), 5-15.

Woods, John (1992). "Who Cares About the Fallacies?", in Frans H. van Eemeren, Rob Grootendorst, J. Anthony Blair and Charles A. Willard (eds.), Argumentation Illuminated, Amsterdam: Sic-Sat, 23-48.

Woods, John and Walton, Douglas (1989). Fallacies: Selected Papers, 1972-1982, Dordrecht: Foris.

\section{JOHN WOODS}

DEPARTMENT OF PHILOSOPHY

THE UNIVERSITY OF LETHBRIDGE

440I UNIVERSITY DRIVE

LETHBRIDGE, ALBERTA

$T / K 3 M 4$ 